Aim of the study: The aim of the study was to develop standards for the prefabrication of free microvascular flaps in an animal model, followed by their application in clinical practice, and quantitative/qualitative microscopic assessment of the extent of development of a new microvascular network.

Material and methods: The study was carried out in 10 experimental pigs. As the first stage, a total of 20 prefabricated flaps were created using polytetrafluoroethylene (PTFE) as a support material, placed horizontally over an isolated and distally closed vascular pedicle based on superficial abdominal vessels. After completing the animal model study, one patient was selected for the grafting of the prefabricated free flap. Results: All 20 free flaps prefabricated in the animal model were analyzed microscopically, exhibiting connective tissue rich in fibroblasts and small blood vessels in the porous areas across the entire thickness of the PTFE element. Conclusions: Flap prefabrication is a new and fast developing reconstruction technique. The usefulness of prefabrication techniques and their status in reconstructive surgery still needs to be investigated experimentally and clinically. The method based on prefabricated free flaps is the first step towards anatomical bioengineering that will make it possible to replace missing organs with their anatomically perfect equivalents.

Key words: prefabrication techniques, reconstructive surgery, free flaps.

\section{The use of prefabrication technique in microvascular reconstructive surgery}

\author{
Łukasz Krakowczyk, Adam Maciejewski, Cezary Szymczyk, Janusz Wierzgoń, \\ Ryszard Szumniak, Piotr Jędrzejewski, Maciej Grajek, Mirosław Dobrut, \\ Rafał Ulczok, Stanisław Półtorak
}

Department of Oncological and Reconstructive Surgery, Center of Oncology Maria Sklodowska-Curie Institute of Oncology, Branch Gliwice, Poland

\section{Introduction}

Oncologic reconstructive and microvascular surgery involves a range of complex and often multi-stage techniques aimed at restoring the structure of soft tissues, bones, blood vessels and nerves previously removed along with the cancerous tumour. The aim of reconstructive surgical procedures is to achieve anatomical, topographical, functional and aesthetic effects which approximate as closely as possible normal physiological conditions. Microsurgery has already defined and investigated the suitability of several hundred free tissue flaps (harvested from distant sites of the body) which can be routinely used for elective reconstructive surgery [1, 2]. In 1964, Nakayama was the first to use a tissue graft based on vascular microanastomoses, which triggered a rapid development of microvascular reconstructive surgery [3].

Despite multiple benefits of using conventional microvascular flaps, there is as yet no perfect flap with individually modifiable characteristics that would ensure optimum functional and visual effects. Great hopes are currently being pinned on the prefabrication of microvascular flaps, since the technique allows extremely precise planning and construction of a complex which closely mirrors missing tissues that have previously been resected [4]. The term prefabrication was coined in 1982 by Shen et al. who described the technique of free flap formation based on a specially prepared vascular pedicle implanted under the selected skin flap fragment [5].

Flap prefabrication refers to the precise customized construction and remodelling of the flap in the donor site. The technique involves the preparation of exactly defined amounts of different flap tissues, followed by flap customization to match the specific needs of the defect to be corrected. A combination of conventional plastic surgery techniques and tissue engineering procedures make prefabrication one of the most promising areas of plastic surgery. The suitability of these techniques and their status in reconstructive surgery still need verification in experimental and clinical investigations [6]. Flap prefabrication techniques require extreme precision, as each inaccuracy, slight as it may be, carries the risk of partial or complete necrosis of the flap, secondary separation of its components or infection. Consequently, introduction of these techniques into clinical practice, just like any modifications of existing methods, must be preceded by experimental studies in animal models in compliance with international laws in place. The studies must provide unequivocal documented evidence for the efficacy and safety of microsurgical reconstruction techniques under investigation $[7,8]$.

\section{Aim of the study}

The aim of the study was to develop standards for the prefabrication of free microvascular flaps in an animal model, followed by their application in clinical practice, and quantitative/qualitative microscopic assessment of the extent of development of a new microvascular network. 


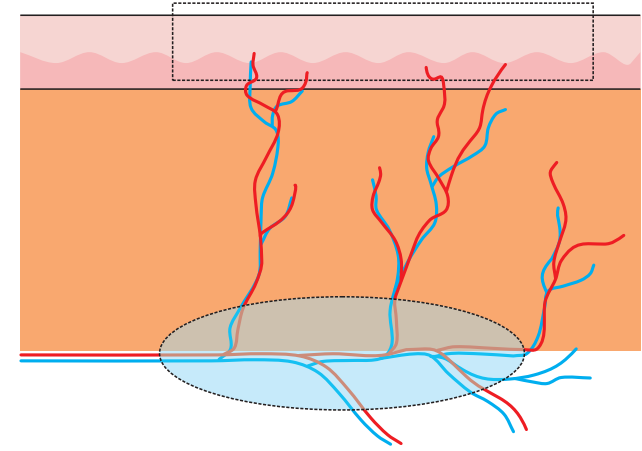

pedicle with fascia or small cuff muscle dissected out

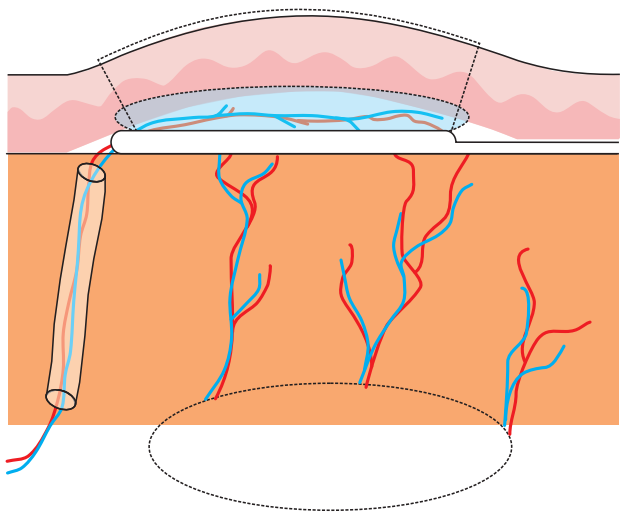

goretex tubing or silicon sheed around proximal pedicle to facilitate later flap harvest
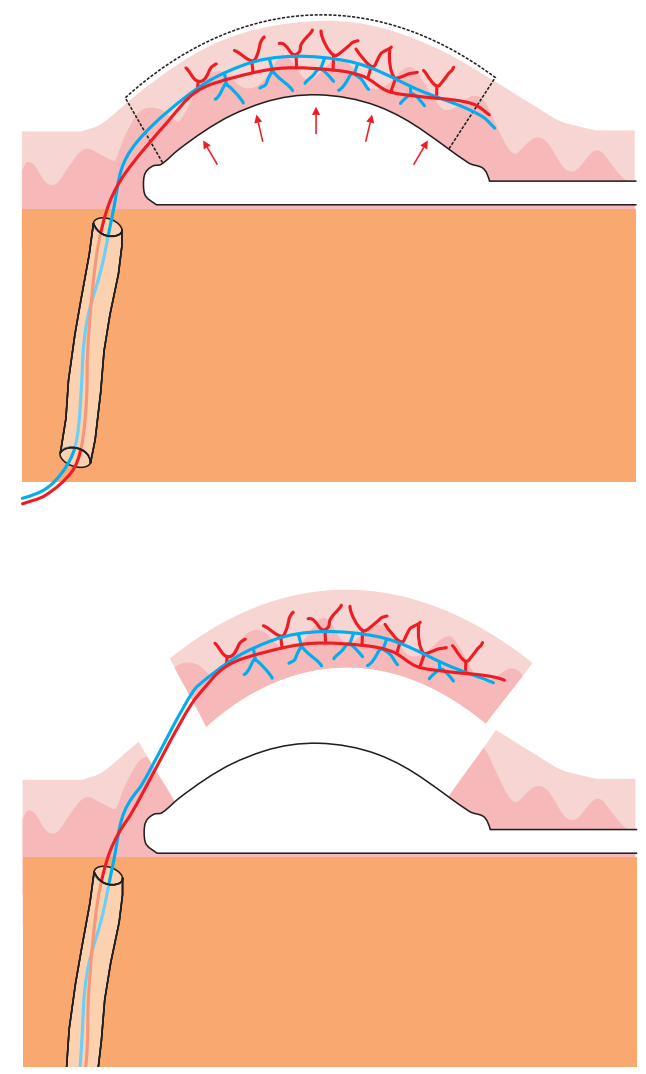

Fig. 1. Prefabrication technique desired skin flap

muscle

deep vascular pedicle

vascular pedicle placed on top of tissue expander

expansion started 1 week and continued for 8 weeks

tissue expander fully expanded at 8 weeks

neovascularization od skin and capsule around tissue expander

prefabricated flap raised with tissue expander of capsule

goretex around proximal pedicle facilitates flap dissection 


\section{Material and methods}

Prior to the application of prefabrication techniques in the Department of Oncologic and Reconstructive Surgery of the Oncology Centre, IMSC Institute in Gliwice, an experimental study was conducted in an animal model. The study was carried out in 10 experimental pigs. As the first stage, a total of 20 prefabricated flaps were created using polytetrafluoroethylene (PTFE) as a support material, placed horizontally over an isolated and distally closed vascular pedicle based on superficial abdominal vessels. Before commencing the surgical procedure each of the animals was anaesthesized with sodium thiopental. All the animals also received prophylactic antibiotic therapy for 24 hours. In view of the activities involved in the process of flap prefabrication, the experiment was scheduled to take place in three stages. An 8-week interval between stages 1 and 2 was sufficient for the process of angiogenesis, formation of the vascular network and adequate vascularization of prefabricate components. Another stage (stage 3 ) was conducted a week after the completion of stage 2 in order to harvest tissue samples for macroscopic and histological analysis. The prefabrication technique is presented in detail in Fig. 1. The operating material, consisting of 20 prefabricated flaps, was fixed in 10\% buffered formalin. Slices were taken and processed in the tissue processor, following which they were embedded in paraffin. $4 \mu \mathrm{m}$ slices were stained with hematoxylin-eosin and then assessed under the light microscope.

After completing the animal model study, one patient was selected for the grafting of the prefabricated free flap. The patient, suffering from cancer of the nose, was not eligible for any other reconstructive surgery methods following complete surgical resection of the nose. A prefabricated vascularized flap was created on the patient's left thigh, on the descending branch of the deep circumflex femoral artery. A total of five PTFE elements implanted in the subcutaneous tissue of the left thigh were used as a support material. After eight weeks a prefabricated free flap was prepared and then transferred to the recipient site (Fig. 2).

\section{Results}

According to the method proposed by Steinhart, Walton and Brown, and Cane [9], the microvascular network overgrows the perforated alloplast and naturally creates a vascularized support element. Following the integration of the alloplast with the vascular network the vascularized graft in the form of a prefabricated microvascular flap can be used as a customized free flap. All 20 free flaps prefabricated in the animal model were analyzed microscopically, exhibiting connective tissue rich in fibroblasts and small blood vessels in the porous areas across the entire thickness of the PTFE element. Numerous foreignbody giant cells were observed in the immediate vicinity of the support material, forming occasional granulomas. Also, there were areas with abundant infiltrations from lymphoid cells, neutrophils granulocytes and eosinophil granulocytes. None of the preparations under analysis revealed the presence of fibrous capsule around the board or collagen fibres in the open spaces. The final outcome of the surgical procedure, as well as functional and aesthetic effects of using a prefabricated free flap in a patient who has had complete surgical resection of the nose, is presented in Fig. 3.
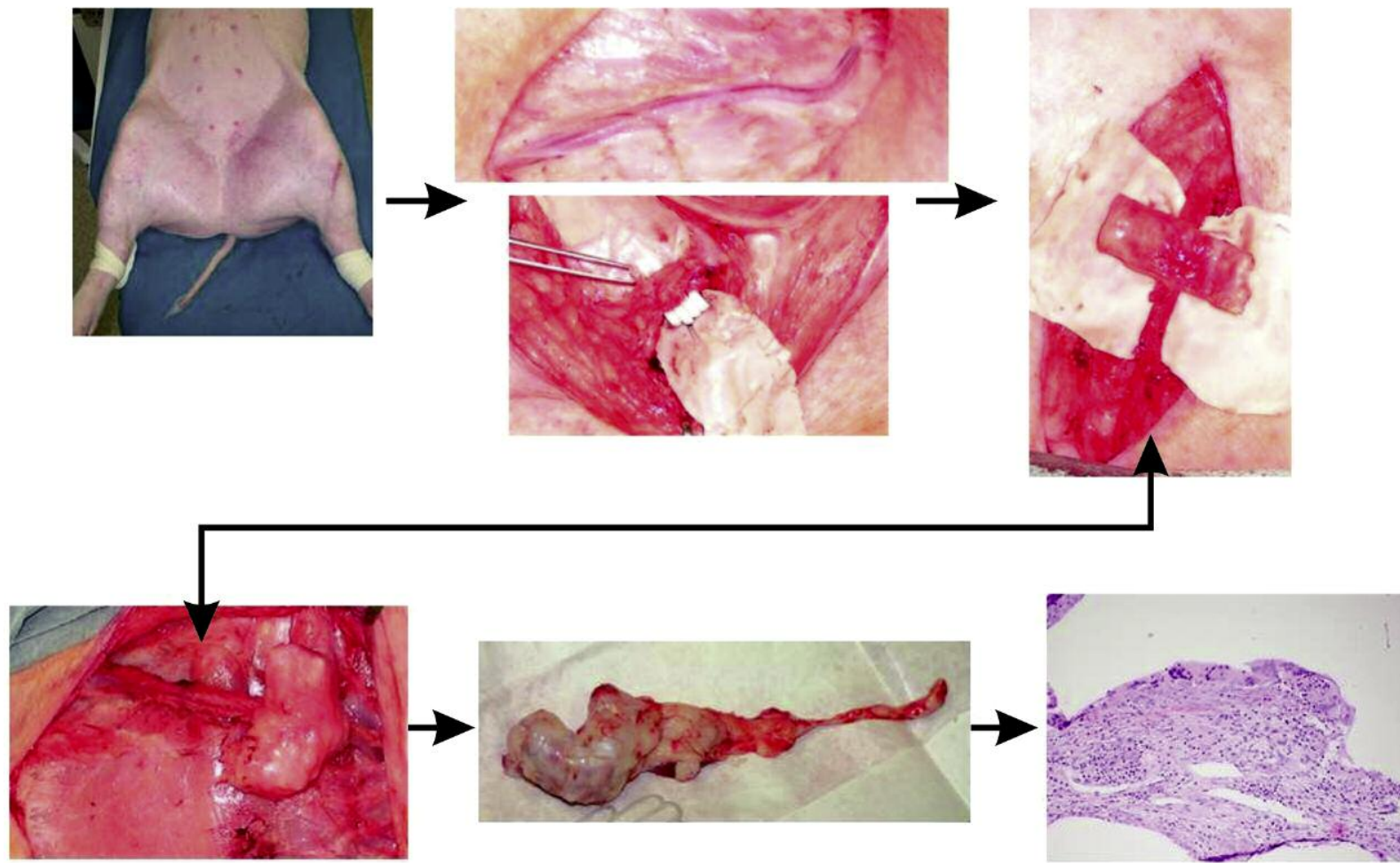

Fig. 2. Experimental data on animal model. 

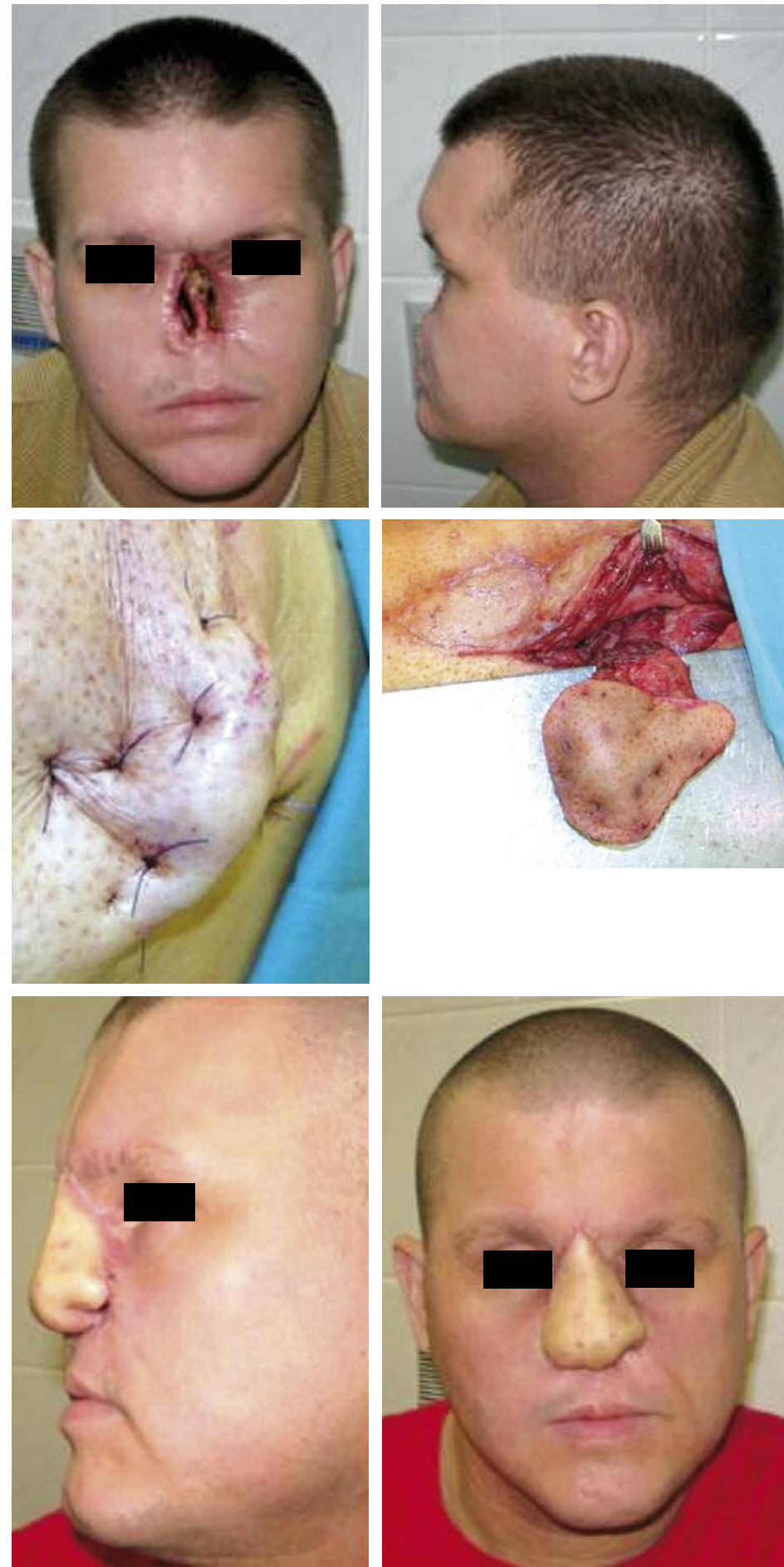

Fig. 3. Aesthetic and functional effect after use of prefabrication free flap. Flap prefabrication starts with introduction of a vascular pedicle (descending branch of the deep femoral artery) to a desired donor tissue that on its own does not possess an axial blood supply. After a period of neovascularization of at least 8 weeks, this donor tissue can be transferred to the recipient defect based on the newly acquired axial vasculature
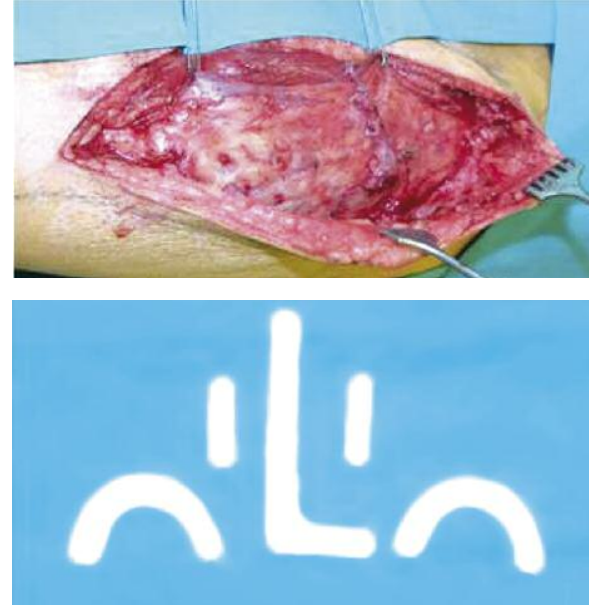

\section{Discussion}

Reconstructive surgery is increasingly searching for new methods that would allow precise and customized design and fabrication of tissue complexes to achieve optimum postoperative effect and, at the same time, ensure reasonably limited functional and aesthetic defects in the donor sites. Even though conventional reconstructive microsurgery techniques are successfully used for correcting the majority of postresective defects within the head and neck area, there are currently no standards applicable to the reconstruction of the nose and other structures of the middle section of the face, the trachea and other large three-dimensional skin areas, while the final effects of reconstruction procedures are usually unsatisfactory.

Most reports published worldwide on problems associated with the prefabrication of artificial microvascular complexes describe experiences with using double-component pedicled flaps prefabricated on small laboratory animals (rats, rabbits) $[8,10,11]$. They have proven beyond doubt that the process of neovascularization carries the possibility of designing simple connections between blood vessels and tissues. The procedure has already been used several times in humans for the reconstruction of defects located in the head and neck region. Moreover, isolated case reports seem to corroborate the claim that alloplastic materials implanted into the developing vascular network can be successfully employed in reconstructive surgery as microvascular support elements.

Reconstruction of the complex structures of the nose, involving nasal bones, cartilage and soft tissues, continues to present some of the most unique challenges to the reconstructive surgeon $[1,12]$. There are as yet no algorithms or standards for nasal reconstruction. Reconstructive surgery of the nose usually involves complex multi- 
stage procedures with prelaminated radial flaps that are combined with non-vascularized grafts of ear or rib cartilage, and additionally covered with rotational frontal flaps. The complexes are, however, very difficult to obtain and the technique requires a high degree of precision. The total duration of all surgical procedures often exceeds 4 years, while the final functional and visual effect is largely unpredictable. Therefore, attempts are constantly being made to find solutions allowing faster, simpler and better nasal reconstruction. Theoretically, if a framework for the nasal root, columella and alae could be precisely modelled out of an artificial material, then transformed into a vascularized structure additionally covered with an inner surface restoring nasal ducts, transposed to the defect site and covered with a frontal flap, the reconstruction procedure would be reduced to just two stages and would yield a very good and predictable final effect [13-15].

Flap prefabrication is a new and fast developing reconstruction technique. The usefulness of prefabrication techniques and their status in reconstructive surgery still needs to be investigated experimentally and clinically. Flap prefabrication techniques call for extreme precision because even the slightest inaccuracy carries the risk of partial or complete necrosis of the flap, secondary separation of its components or infection. Introduction of these techniques into clinical practice, just like any modifications of existing methods, must be preceded by experimental studies in animal models in compliance with international laws. The studies must provide indisputable evidence for the efficacy and safety of microsurgical reconstruction techniques under investigation $[9,16]$.

Good effects obtained to date by the Reconstructive and Microvascular Surgery Team at the Oncology Institute in Gliwice (three-year symptom-free survival rate of $85 \%$ in a group of over 300 patients suffering from locally advanced cancer of the oral cavity and throat) validate and legitimize the extension of the arsenal of available reconstruction surgery techniques with prefabricated flaps in order to improve the functional and aesthetic outcome, and expand the qualification criteria for these therapeutic options. The method based on prefabricated free flaps is the first step towards anatomical bioengineering that will make it possible to replace missing organs with their anatomically perfect equivalents [17]. Although a considerable body of experimental research and broadly understood cooperation between medical centres are still necessary, it seems quite likely that tissue bioengineering will one day make routine use of the vascular foundations of prefabrication techniques.

The authors declare no conflict of interest.

\section{References}

1. Shen TY. Vascular implantation into skin flaps:experimental study and clinical application:a preliminary report. Plast Reconstr Surg 1981; 68: 404-10.

2. Erol OO. The transformation of a free skin graft into a vascularized pedicled flap. Plast Reconstr Surg 1976; 58: 470-7.

3. Tan BK, Chen HC, He TM, Song IC. Flap prefabrication - the bridge between conventional flaps and tissue-engineered flaps. Ann Acad Med Singapore 2004; 33: 662-6.
4. Atabey A, McCarthy E, Manson P, Vander Kolk CA. Prefabrication of combined composite (chimeric) flaps in rats. Ann Plast Surg 2000; 45: 581-7.

5. Walton R, Brown R, Zhang L. Creation of a vascularized alloplastic unit for composite reconstruction. Plast Surg Forum 1987; 56: 32.

6. Staudenmaier R, Hoang TN, Kleinsasser N, Schurr C, Frölich K, Wenzel MM, Aigner J. Prefabrication of large fasciocutaneous flaps using an isolated arterialised vein as implanted vascular pedicle. J Reconstr Microsurg 2004; 20: 555-64.

7. Baudet J, Pelissier P, Casoli V. 1984-1994: Ten years of skin flaps. Prefabricated flaps. Ann Chir Plast Esthet 1995; 40: 597-605.

8. Abbase EA, Shenaq SM, Spira M, el-Falaky MH. Prefabricated flaps: experimental and clinical review. Plast Reconstr Surg 1995; 96: 1218-25.

9. Khouri RK, Upton J, Shaw WW. Principles of flap prefabrication. Clin Plast Surg 1992; 19: 763-71.

10. Pribaz JJ, Maitz PK, Fine NA. Flap prefabrication using the "vascu lar crane" principle: an experimental study and clinical application. Br J Plast Surg 1994; 47: 250-6.

11. Can Z, Apaydin I, Erçöçen AR, Demirseren ME, Sabuncuo?lu B. Prefabrication of a high-density porous polyethylene implant using a vascular induction technique. Ann Plast Surg 1998; 41: 264-9.

12. Ozdemir R, Kocer U, Tiftikcioglu YO, Karaaslan O, Kankaya Y, Cuzdan S, Baydar DE. Axial pattern composite prefabrication of highdensity porous polyethylene: experimental and clinical research. Plast Reconstr Surg. 2005; 115: 183-96.

13. Guo L, Pribaz JJ. Clinical flap prefabrication. Plast Reconstr Surg 2009; 124: 340-50.

\section{Adres do korespondencj}

dr n. med. Łukasz Krakowczyk

Department of Oncological and Reconstructive Surgery

Center of Oncology - Maria Sklodowska-Curie

Institute of Oncology, Branch Gliwice

Wybrzeże Armii Krajowej 15

44-101 Gliwice, Poland

tel. +48322788417

fax +48322788417

e-mail: lukaszkrakowczyk@wp.pl

Submitted: $\quad 11.12 .2011$

Accepted: $\quad$ 18.04.2012 